Chirurg 2018 $89: 158$

https://doi.org/10.1007/s00104-018-0600-y

Online publiziert: 24. Januar 2018

(c) Springer Medizin Verlag $\mathrm{GmbH}$, ein Teil von Springer Nature 2018

CrossMark

W. Schröder · C. J. Bruns

Klinik für Allgemein-, Viszeral- und Tumorchirurgie, Universitätsklinik Köln, Köln, Deutschland

\title{
Einfluss der Zentralisierung auf das Outcome der Ösophagusperforation
}

und „High-volume“-Zentren (< und $\geq 20$ onkologische Resektionen).

Markar SR, Mackenzie H, Wiggins T et al (2018) Influence of national centralization of oesophagogastric cancer on the management and clinical outcome from emergency upper gastrointestinal conditions. Br J Surg 105:113-120. https://doi.org/10. 1002/bjs.10640

Hintergrund und Fragestellung. Für die chirurgische Behandlung des Ösophagus- und Magenkarzinoms ist hinreichend nachgewiesen, dass die Zentralisierung und damit die Zunahme der Fallzahlen in einzelnen Zentren zu einer Reduktion der postoperativen Morbidität und Mortalität führen. Ob die Zentralisierung dieser onkologischen Erkrankungen auch gleichzeitig einen positiven Einfluss auf die Ergebnisse von Notfallbehandlungen am oberen Gastrointestinaltrakt hat, wurde jetzt in einer retrospektiven Studie aus England untersucht.

Methoden. In einer Registeranalyse der Hospital-Episode-Statistics(HES)Datenbank, welche die Patientendaten des National-Health-Systems (NHS) in England sammelt, wurden von 1997 bis 2012 insgesamt 3707 Ösophagusperforationen (OEP), 12.441 inkarzerierte paraösophageale Hernien $(\mathrm{POH})$ und 56.822 perforierte peptische Ulzera (PPU) dokumentiert und ausgewertet. Von den Autoren wurde die OEP als Notfall mit niedriger, die $\mathrm{POH}$ als Notfall mit durchschnittlicher und die PPU als Notfall mit hoher Inzidenz klassifiziert. Primärer Zielparameter für diese drei Krankheitsbilder war die 30- und 90-Tage-Mortalität unterteilt für „Low-“
Ergebnisse. In dem Beobachtungszeitraum von 15 Jahren konnte analog $\mathrm{zu}$ den onkologischen Resektionen ein deutlicher Anstieg zur Behandlung dieser drei Notfällen in „High-volume“-Zentren beobachtet werden, für die OEP war er mit $26,9 \%$ am höchsten. Die 30-Tage-Mortalität lag für die OEP bei $28,5 \%$, für die PPU bei $24,1 \%$ und für die $\mathrm{POH}$ bei 7,0\%. Für die OEP war eine Reduktion der Sterblichkeit beim Management in einem „High-volume“-Zentrum nachzuweisen (30-Tage-Mortalität: Hazard Ratio [HR] 0,58 mit 95\%-Konfidenzintervall[CI] von 0,45 und 0,74 ; 90 -Tage-Mortalität: HR 0,62 mit $95 \%$-CI von 0,49 und 0,77). Die Schwelle für eine Senkung der Mortalität nach OEP wurde mit 5 Fällen pro Jahr berechnet. Die Mortalität der POH und des PPU wurde dagegen nicht durch die jährliche Fallzahl des behandelnden Krankenhauses beeinflusst.

Fazit. Diese große Untersuchung aus England zeigt einen deutlichen Trend, Notfälle des oberen Gastrointestinaltraktes in spezialisierten Zentren $\mathrm{zu}$ behandeln. Dieser Anstieg wird von den Autoren als passiver Nebeneffekt der intendierten Zentralisierung der Behandlung des Ösophagus- und Magenkarzinoms bewertet. Allerdings wirkt sich diese Zentralisierung bei den Behandlungsergebnissen nur bei der relativ seltenen Ösophagusperforation aus - einem Notfall, welcher in hohem Maße von einem frühzeitigen Therapiebeginn, einer ausgewiesenen endoskopischen und chirurgischen Expertise und der dafür notwendigen Infrastruktur eines 24-Stunden-Service profitiert.

\section{Korrespondenzadresse}

Prof. Dr. W. Schröder, FACS, FEBS

Klinik für Allgemein-, Viszeral- und Tumorchirurgie, Universitätsklinik Köln Kerpener Str. 62, 50937 Köln, Deutschland wolfgang.schroeder@uni-koeln.de

Interessenkonflikt. W. Schröder und C. J. Bruns geben an, dass kein Interessenkonflikt besteht. 